\title{
Principle and Approach for Determining the Reasonable Cross-Section of the Solid-Web Double-beam Component
}

\author{
Aifeng Li \\ Taiyuan Heavy Industry CO,.LTD,NO.53 Yuhe Street,Wanbailin District,Taiyuan City,Shanxi \\ Prov, China 030024 \\ liaifeng120@163.com
}

Keywords: Excavator Steel structure Optimization design Dipper handle

\begin{abstract}
Solid-web double-beam component is widely used in the dipper handle of excavator, whose mechanical behaviour belongs to compression-bending member and mainly satisfies the strength requirement. In this paper, it starts from the design principles of steel structure and elastic theory, determines the dimension of the main cross-section and the reverse tube under guidance of optimization, and provides initial data for the detailed design. The method has common direct sense.
\end{abstract}

\section{Foreword}

Solid-web double-beam component is widely used in the dipper handle of mechanic excavators, whose mechanical behavior belongs to compression-bending member and mainly satisfies the strength requirement. In initial design, how to use its basic data, determine its reasonable cross-section dimension and provide initial data for the detailed design, designers are got into trouble. Thus, determine the reasonable dimension of the main cross-section from the design principles of steel structure under guidance of optimization and taking the example of excavator dipper handle.

To determine the cross-section of composite beam, the overall design requirement of components shall be comprehensively considered to meet the requirement of strength and economy. It is the issue of optimization.

\section{Mechanical model}

\subsection{Requirements for strength}

Where the ratio of length $l$ and height $\mathrm{h}$ of a component $l / h>5$, it can be regarded as a beam. According to strength requirement of beam,

$$
\sigma=\frac{M}{W}+\frac{N}{A} \leq[\sigma]
$$

\subsection{Function expression of beam dead weight}

Beams with wide universality in components are composite I beams and composite box beams. Excavator movable arm belongs to composite box beam, generally composed of flange plate, web plate and web stiffener. Gross mass of beam $G$ is the summation of flange plate weight $G_{1}$, web plate weight $\mathrm{G}_{2}$, web stiffener weight $\mathrm{G}_{3}$ and connecting weld joint weight $\mathrm{G}_{4}$.

$$
\begin{aligned}
& G=G_{1}+G_{2}+G_{3}+G_{4}=G_{1}+n_{1} G_{2} \\
& n_{1}=\left(G_{2}+G_{3}+G_{4}\right) / G_{2}, \quad n_{1} \geq 1
\end{aligned}
$$




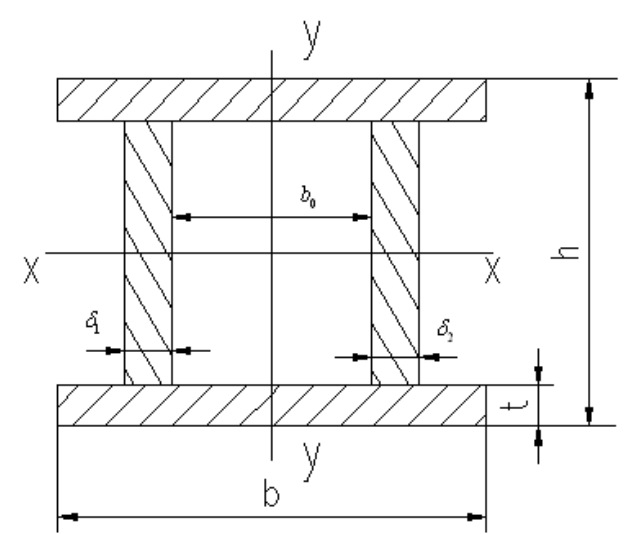

Fig.1 Cross-section of box beam

$$
\begin{aligned}
& G_{1}=2 A_{1} \cdot l \cdot r \\
& G_{2}=h \sum \delta_{i} \cdot l \cdot r \\
& G=\left(2 A_{1}+n_{1} h \sum_{i=1}^{m} \delta_{i}\right) l \cdot r
\end{aligned}
$$

Wherein, ${ }^{A_{1}}$ : Cross-sectional area of a flange plate

$$
\sum \delta_{i} \text { : Total thickness of web plate }
$$

$n_{1}$ : Structural coefficient of web member

$l$ : Length of dipper handle, determined in population parameter design $r$ :Steel density, $r=7.8 \mathrm{t} / \mathrm{m} 3$ 。

(1) Determine formula $G_{\sigma}$ of beam dead weight according to strength requirement:

$$
\begin{aligned}
& I_{\sigma}=\frac{W h}{2} \approx 2 A_{1}\left(\frac{h}{2}\right)^{2}+\frac{h^{3} \sum \delta_{i}}{12} \\
& A_{1}=\frac{W}{h}-\frac{h \sum \delta_{i}}{6} \\
& G_{\sigma}=\left(\frac{2 W}{h}-\frac{h \sum \delta_{i}}{3}+n_{1} h \sum \delta_{i}\right) l \cdot r=\left[\frac{2 W}{h}+\left(n_{1}-\frac{1}{3}\right) h \sum \delta_{i}\right] l \cdot r
\end{aligned}
$$

To get $\min G_{\sigma}$, supposing $\frac{d G_{\sigma}}{d h}=0$, that is

$$
\begin{aligned}
& h_{\sigma}=k_{\sigma} \sqrt{\frac{W}{\sum \delta_{i}}} \\
& k_{\sigma}=\sqrt{\frac{2}{n_{1}-\frac{1}{3}}}=\frac{\sqrt{2}}{\sqrt{n_{1}-\frac{1}{3}}}
\end{aligned}
$$

From expression (7), $\sum \delta_{i} h_{\sigma}^{2}=k_{\sigma}^{2} W$, thus

$$
W=\frac{\sum \delta_{i} h_{\sigma}^{2}}{k_{\sigma}^{2}}
$$


Dipper handle of mechanical excavator plays the role of stress and bending mainly, whose torsion is sustained by reverse tube. Thus,

$$
\begin{aligned}
& \frac{N}{A}+\frac{M_{x}}{W_{x}} \leq[\sigma] \\
& A=2 A_{1}+\sum \delta_{i} h
\end{aligned}
$$

Wherein, $A$ : Effective area of cross section

$N$ : Axial force acting on cross section

$W_{x}$ : Flexural modulus of cross section $\mathrm{x}$ axis

$M_{x}:$ x axis bending moment of cross section

From formula (5) and (8), determine functional relationship $A=f\left(h_{\sigma}, \delta_{i}\right)$. Through combination, get

$$
A=\frac{2 \sum \delta_{i} h_{\sigma}\left(3+k_{\sigma}^{2}\right)}{3 k_{\sigma}^{2}}
$$

Make substitution (13) into (12) and (14),

$$
\frac{\frac{N}{2 \sum \delta_{i} h_{\sigma}\left(3+k_{\sigma}^{2}\right)}}{3 k_{\sigma}^{2}}+\frac{M}{\frac{\sum \delta_{i} h_{\sigma}^{2}}{k_{\sigma}^{2}}} \leq[\sigma]
$$

Then cross section $h_{\sigma}$, determined by external load and basic parameters, can be obtained.

$$
\begin{aligned}
& \text { If } i=2 \text {, and } t=\delta_{1}=\delta_{2}=\delta \\
& \qquad h_{\sigma}=\frac{3 k_{\sigma}^{2} \cdot N \pm \sqrt{9 k_{\sigma}^{4} N^{2}+32 \delta\left(3+k_{\sigma}^{2}\right)^{2} \cdot k_{\sigma}^{2} \cdot[\sigma] \cdot M_{x}}}{8 \delta\left(3+k_{\sigma}^{2}\right)[\sigma]}
\end{aligned}
$$

$A_{1}$ can be calculated by making substitution of $h_{\sigma}$ into (8) and (5).

\section{Determine the reasonable cross section of hopper handle}

\subsection{Determine the center width between two hopper handles}

Inside width of hopper handle is determined by the distance between hopper handle and hopper hinge point which exerts a tremendous effect on hopper intensity. Thus, the center width of two hopper handles can be determined by hopper hinge point distance.

Hinge point between hopper handle and hopper is located the rear wall of hopper. Thickness of rear wall is much smaller than width and length of rear wall, simplified as simply support overhanging beam structure of both ends. Normal component of force of load (Including soil counter-force) acts at hopper rear wall and is considered as uniformly distributed load, as shown in Fig 2.

$$
\begin{aligned}
& R_{A}=R_{B}=q\left(\frac{l}{2}+a\right), \text { shearing force } \mathrm{Q}, \\
& 0 \leq z \leq a, \quad Q=-q z \\
& a \leq z \leq a+l, \quad Q=R_{A}-q z=q\left(\frac{l}{2}+a\right)-q z=\frac{q l}{2}\left(1+\frac{2 a}{l}-\frac{2 z}{l}\right) \\
& a+l<z \leq l+2 a, Q=R_{A}+R_{B}-q z=q\left(\frac{l}{2}+a\right)+q\left(\frac{l}{2}+a\right)-q z=q(l+2 a-z)
\end{aligned}
$$




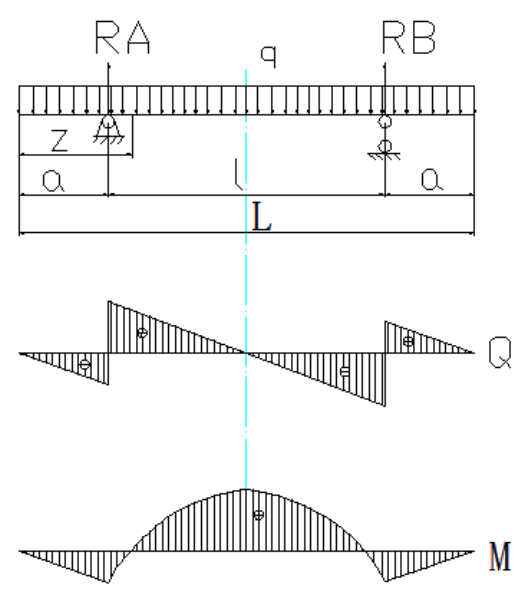

Fig.2 Shearing force and bending moment

$$
\begin{aligned}
& 0 \leq z \leq a, M_{I}=-\frac{q z^{2}}{2} \\
& a \leq z \leq a+l, \\
& M_{I I}=R_{A}(z-a)-\frac{1}{2} q z^{2}=q\left(\frac{l}{2}+a\right)(z-a)-\frac{1}{2} q z^{2}=\frac{q l^{2}}{2}\left[\left(1+\frac{2 a}{l}\right)\left(\frac{z}{l}-\frac{a}{l}\right)-\frac{z^{2}}{l^{2}}\right]
\end{aligned}
$$

To evaluate $M_{\text {II Max }}$

$$
\begin{aligned}
& \frac{d M_{\mathrm{II}}}{\mathrm{dz}}=\frac{d}{d_{z}}\left\{\frac{q l^{2}}{2}\left[\left(1+\frac{2 a}{l}\right)\left(\frac{z}{l}-\frac{a}{l}\right)-\frac{z^{2}}{l^{2}}\right]\right\}=0, \quad \text { that is } \\
& \mathrm{Z}=\frac{l+2 a}{2}=a+\frac{l}{2} \\
& M_{\mathrm{II} \operatorname{Max}}=\frac{q l^{2}}{2}\left[\frac{1}{4}-\frac{a^{2}}{l^{2}}\right]
\end{aligned}
$$

Provided that the beam is uniform cross section beam, then $M_{1}=M_{\mathrm{II} M a x}$,

$$
\text { That is } \frac{q a^{2}}{2}=\frac{q l^{2}}{2}\left[\frac{1}{4}-\frac{a^{2}}{l^{2}}\right], l^{2}=8 a^{2}, l=2 \sqrt{2} a
$$

Center distance between two hopper handles

$$
a=L /(2 \sqrt{2}+2)
$$

Suppose $l+2 a=2 \sqrt{2 a}+2 a=L=4.86 m$ '(Inside hopper width)

$$
a=4.86 /(2 \sqrt{2}+2)=1.0065 \approx 1 m \quad l=2.8469 \approx 2.847 m,
$$

That is, center width between two hoppers is $2.847 \mathrm{~m}$. Actual center width between two hoppers is $2.7 \mathrm{~m}$ 。

Error: $\eta_{1}=\frac{2.847-2.7}{2.7}=5.4 \%$; 


\subsection{Cross-sectional design of hopper handle}

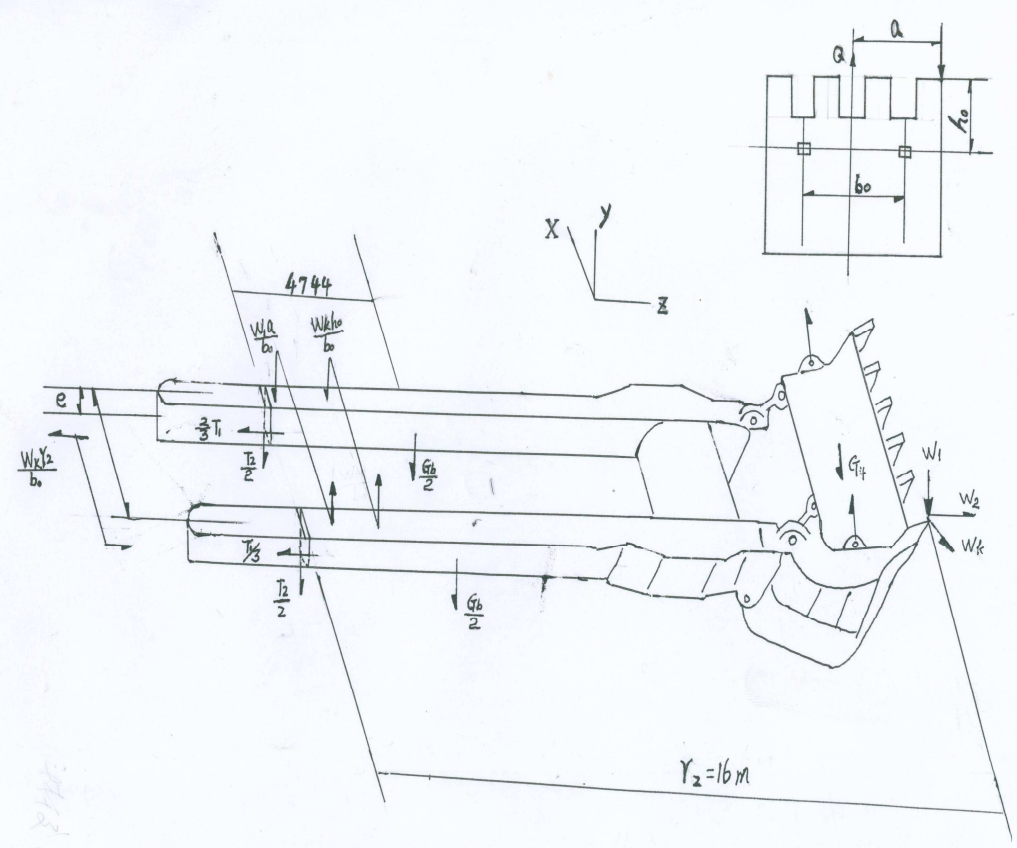

Fig.3: Force diagram of hopper handle

Hopper handle design ${ }^{[1]}$ includes structure form of hopper handle, hopper handle length, cross-sectional design and cross-sectional design of reverse tube.

Hopper handle is push-type gear rack, solid-web double-beam component, added with ribbed slab along its cross section direction, welded cylinder at front hopper handle next to hopper used for anti-torque.

\section{(1)Assumed conditions}

Assumed conditions include the following.

(1)Considering hopper body stiffness is much larger than hopper handle stiffness. Thus hopper handle shall be regarded as cantilever mechanic model[30];

(2) Suppose uneven two loaded handle hoppers. And suppose hopper handle at one side is sustained by $2 \mathrm{~T} 1 / 3$ and another side T1/3.

(3) Take the hopper handle sustaining the larger load as computing object.

(4)For that handle hopper, $\sigma_{s}=700 \mathrm{Mpa}$;

(5) $[\sigma]=0.85 \sigma_{s}$, impact coefficient 2.5 and safety coefficient 1.2 1.5。

$$
[\sigma]=\frac{700}{2.5 \times(1.2 \sim 1.5)}=233 \sim 186 \mathrm{Mpa}
$$




\section{(2)Known data}

Tangential resistance $\mathrm{W} 1=1325 \mathrm{KN}$, normal resistance $\mathrm{W} 2=1127 \mathrm{KN}, \mathrm{b} 0=2692 \mathrm{~mm}, \mathrm{a}=2.615 \mathrm{~m}$; Crossrange excavating resistance $\mathrm{Wk}=113 \mathrm{KN}$, lifting force $\mathrm{Q}=2890 \mathrm{KN}$, h0 $=4800 \mathrm{~mm}$; Pushing force $\mathrm{T} 1=1127 \mathrm{KN}$; radial force $\mathrm{T} 2=179 \mathrm{KN}, \mathrm{r} 2=16 \mathrm{~m}$; Swing arm height of pushing axis: $2380 \mathrm{~mm}$; contact length of hopper handle and swing arm: $2380 / \sin 45^{\circ}=3366 \mathrm{~mm}$; Diagonal contact length: 4744mm;

Downward component forces of vertical hopper handle center line:

$$
\begin{aligned}
& p_{1}=\frac{w_{1} a}{b_{0}}=\frac{1325 \times 2.615}{2.692}=1287 \mathrm{KN}, \\
& p_{2}=\frac{w_{k} h_{0}}{b_{0}}=\frac{113 \times 4.8}{2.692}=202 \mathrm{KN}, \\
& p_{3}=T_{2} / 2=179 / 2=90 \mathrm{KN}, \\
& P=p_{1}+p_{2}+p_{3}=1579 \mathrm{KN}
\end{aligned}
$$

Bending moment $\mathrm{M}=\mathrm{PL}, \mathrm{L}$ is the distance between cross section and pushing axis.

Resultant forces along hopper handle center line:

$$
N=\frac{2}{3} T_{1}+\frac{w_{k} r_{2}}{b_{0}}=\frac{2}{3} \times 1127+\frac{113 \times 16}{2.692}=751.3+672=1423 K N
$$

\begin{tabular}{|c|c|c|c|c|c|c|c|}
\hline & \multicolumn{7}{|c|}{ Computing position $\mathrm{I}: \mathrm{L}=5 \mathrm{~m}$ exterior cross section from pushing axis } \\
\hline & \multicolumn{3}{|c|}{$[\sigma]=200 \mathrm{Mpa}$} & \multicolumn{4}{|c|}{$[\sigma]=185 \mathrm{Mpa}$} \\
\hline & $\delta=25$ & $\delta=50$ & $\delta=75$ & $\delta=25$ & $\delta=38$ & $\delta=50$ & $\delta=75$ \\
\hline \multirow{5}{*}{$\begin{array}{l}\text { Hopper } \\
\text { handle } h_{\sigma}(m)\end{array}$} & 1.4 & 0.98 & 0.79 & 1.455 & 1.173 & 1 & 0.83 \\
\hline & \multicolumn{7}{|c|}{ Computing position $I I$; $L=11.3 m$ exterior cross section from pushing axi } \\
\hline & 1.46 & 1.19 & 2.16 & 1.76 & 1.62 & 1.62 & 1.32 \\
\hline & \multicolumn{7}{|c|}{ Computing position III: $L=10 \mathrm{~m}$ exterior cross section from pushing axis } \\
\hline & 1.96 & 1.469 & 1.2 & 2.03 & 1.64 & 1.52 & 1.24 \\
\hline
\end{tabular}

\section{(3) Computed result of hopper handle economic height $h_{\sigma}$}

$k_{\sigma}^{[2,3]}=1.52, \quad k_{\sigma}^{2}=2.3104, \quad k_{\sigma}^{4}=5.33794816,3 k_{\delta}^{2}=6.9312,3+k_{\delta}^{2}=5.3104 ;$,

According to formula (12), substituting related data ${ }^{[\sigma]}, \delta, h_{\sigma}$ can be got. Refer to table1:

Table 1 Computed result of hopper result $h_{\sigma}$ :

If $^{[\sigma]}=200 \mathrm{Mpa}, \delta=75 \mathrm{~mm}$, hopper handle root, position close to reverse tube, hopper handle height is $1.2 \mathrm{~m}$;

If $^{[\sigma]}=185 \mathrm{Mpa}, \delta=75 \mathrm{~mm}$, hopper handle root, hopper handle height is $1.24 \mathrm{~m}$; actual hopper handle height: $\mathrm{h}=1219 \mathrm{~mm}$. 


\section{(4) Width of hopper handle}

Width of single hopper handle is determined by the width of pinion mate.

\subsection{Calculation of reverse tube}

Reverse is caused when $\mathrm{T} 1$ and ${ }^{w_{k}} r_{2} / b_{0}$ acting on meshing point between hopper handle and pushing gear is simplified on hopple arm central plane. Function of reverse tube is anti-torque. For easy calculation, regard reverse tube as rectangle uniform section.

(1) Calculating reverse tube side length by allowable shearing stress

Shearing stress can be calculated as per the formula of membrane theory in elasticity mechanics [4].

$$
\tau=\frac{M}{2 A \delta}
$$

Wherein,

M: torque

$\mathrm{M}=1423 \mathrm{KN} \times 0.6 \mathrm{~m}=8.538 \times 108 \mathrm{~N} \cdot \mathrm{mm}$;

A: Average area value encircling cross section outside of reverse tube

$\delta$ : take wall thickness of reverse tube as $\delta=10 \mathrm{~mm}$

Considering $\tau=\frac{M}{2 A \delta} \leq[\tau]$, then $A=\frac{M}{2[\tau] \delta}$; taking $[\tau]=22 M p a$, then

$$
A=\frac{M}{2[\tau] \delta}=\frac{8.538 \times 10^{8}}{2 \times 22 \times 10}=1.94 \times 10^{6} \mathrm{~mm}^{2}
$$

If ratio of long and short sides is $2: 1$, then

Approximate values of long and short side are $2000 \mathrm{~mm}$ and $1000 \mathrm{~mm}$ respectively.

\section{(2) Calculating torsion angle}

$$
\text { Torsion angle: } k=\frac{M S}{4 A^{2} G \delta}
$$

Wherein, S: overall length of tube wall center line;

$G$ :torsional modulus, $G=8.538 \times 10^{8}$ 。

$$
k=\frac{M S}{4 A^{2} G \delta}=\frac{8.538 \times 10^{8} \times 5960}{4 \times 4 \times 10^{12} \times 8.1 \times 10^{4} \times 10}=4 \times 10^{-6} \text { degree }
$$

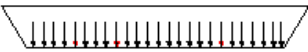

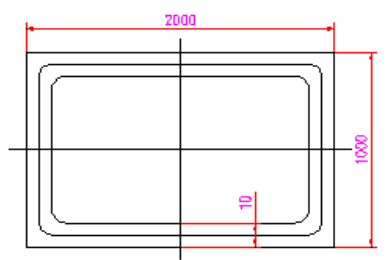

Fig.4 Membrane figure of thin-walled reverse tube 


\section{Conclusion}

Arrive at the following conclusions from computed results:

(1) Center width of two hopper handles is $2.85 \mathrm{~m}, 0.15 \mathrm{~m}$ larger than the actual value;

(2) When $[\sigma]=200 \mathrm{Mpa}, \delta=75 \mathrm{~mm}$, hopper handle root and hopper handle height is $1.2 \mathrm{~m}$; actual hopper handle height is $\mathrm{h}=1.220 \mathrm{~m}$ and error is $-1.64 \%$;

When $[\sigma]=185 \mathrm{Mpa}, \delta=75 \mathrm{~mm}$, hopper handle root and hopper handle height is $1.24 \mathrm{~m}$; actual hopper handle height is $\mathrm{h}=1.220 \mathrm{~m}$ and error is $+1.64 \%$.

(3) Simplified mechanic model and computing method has guiding significance to initial design.

\section{References}

[1] S.W.Yan Design of Mechanical Excavator (China machine Press, pp11-83, Beijing China 1991)

[2] W.Z.Cheng Metallic Structure of Cranes (China Communications Press, pp.117-121, Beijing China 1986)

[3] Z.L.Xu Elasticity Mechanics (Higher Education Press, pp.314-331, Beijing China 1990) 